\title{
EPIDERMOLYSIS BULLOSA AND KINDLER SYNDROME - WHERE IS THE BORDER IN ORAL MANIFESTATIONS
}

\author{
Elitsa Deliverska, Assya Krasteva \\ Faculty of Dental Medicine, Department of Oral and Maxillofacial Surgery, \\ Medical University of Sofia
}

\begin{abstract}
Epidermolysis bullosa (EB) represents a rare chronic autoimmune subepidermal vesiculobullous disorder that affects the skin and mucosa and is characterized by blistering and mechanical fragility of the skin. The most recent classification recognizes four major EB groups: intraepidermal EB (Simplex), junctional EB, dermolytic EB (Dystrophic), and mixed EB (Kindler syndrome). Kindler syndrome is a rare genodermatosis with clinical features of trauma-induced blistering, poikiloderma, skin atrophy, mucosal inflammation and varying degrees of photosensitivity. Although Kindler syndrome is classified as a subtype of epidermolysis bullosa, it has distinct clinicopathological and molecular abnormalities. The clinical features of the syndrome have been annotated by different authors but definite criteria to confirm the diagnosis have not yet been generally accepted.
\end{abstract}

Keywords: Epidermolysis bullosa, Kindler syndrome, oral and maxillo-facial signs

\section{INTRODUCTION}

The term epidermolysis bullosa (EB) describes a heterogeneous group of inherited blistering mucocutaneous disorders. Each has a specific defect in the attachment mechanisms of the epithelial cells either to each other or to the underlying connective tissue. Recent advances in the understanding of the clinical epidemiologic, and molecular genetic abnormalities of these conditions have led to the identification of 4 different forms $(3,16,22,23)$.

The four major EB groups include: 1. intraepidermal EB (Simplex); 2. junctional EB; 3. dermolyt-

Address for correspondence:

Elitsa Deliverska

Faculty of Dental Medicine

Department of Oral and Maxillofacial Surgery

Medical University of Sofia

1 Georgi Sofiyski blvd.

1431 Sofia, Bulgaria

phone: +359888949740

e-mail:elitsadeliverska@yahoo.com

Received: January 15, 2015

Accepted: May 21, 2015 ic EB (Dystrophic); and 4. mixed EB (Kindler syndrome) $(22,23)$.

The onset of EB is very early in life, however, severity varies greatly such that milder forms of the disease are often not identified for many years (2).

\section{INTRAEPIDERMAL EPIDERMOLY- SIS BULLOSA \\ Cause}

The EB Simplex subtypes are caused by mutations in the PKP1, DSP, KRT5, KRT14, PLEC1, ITGA6 and genes (22).

\section{Oral signs}

The genes cause intra-epidermal cleavage in the skin and in the oral mucosa. Individuals with EB Simplex exhibit an increased fragility of the oral mucosa and occur most often secondary to trauma or tissue manipulation. Typically oral soft tissue lesions heal without scarring (20).

\section{Dental caries}

Although many of the genes that are known to be causative of EB Simplex, there is a normal tooth formation (22). 


\section{Salivary function}

Salivary function appears to be normal in people with EB. (22).

\section{JUNCTIONAL EPIDERMOLYSIS BULLOSA \\ Cause}

The Junctional forms of EB are caused by mutations in LAMA3, LAMB3, LAMC3, COL17A1, ITG6A, and ITGB4 that are important in basement membrane mediated cell adhesion (22). The proteins transcribed from these genes are important in epithelia cell adhesion in both the oral mucosa and the developing tooth bud.

\section{Oral signs}

Individuals with Junctional forms of EB have an increased fragility of the oral mucosa and do not have significant oral scarring (22).

\section{Dental caries}

Individuals with Junctional EB are at increased risk for developing dental caries and they have marked enamel defects. The presence of extensive pitting over the tooth surface creates non-cleansable areas that are ideal for microbial growth and substrate retention (22).

\section{Salivary function}

Salivary function appears to be normal in most patients with Junctional EB (22).

\section{DERMOLYTIC EPIDERMOLYSIS BULLOSA \\ Cause}

The Dystrophic EB types are caused by mutations in COL7A1 gene that codes for anchoring fibril protein that are located below the basal lamina at the dermal-epidermal basement membrane zone EB (22).

\section{Oral signs}

In individuals with Dystrophic EB the oral manifestations can involve (16,19,22,23):

* increased tissue fragility of oral and perioral mucosa

* oral ulcerations, healing with scarring.

* blister formation and healing with scarring

* ankyloglossia

* restricted oral aperture and microstomia

\section{Dental caries}

The presence of marked oral blistering affected individuals frequently eat slowly and consume relatively soft diets. The loss of normal tongue mobility and obliteration of the oral vestibule decrease the normal food clearance causing additional prolongation of the dental surfaces to potentially cariogenic substrates (22).

\section{Salivary function}

Despite normal salivary secretion in most people with dermolytic EB subtypes, the oral cavity tends to be inoculated with high numbers of bacteria and there tends to be excessive tooth plaque formation that further promote the formation of dental caries (22).

\section{MIXED EPIDERMOLYSIS BULLOSA (KINDLER SYNDROME)}

Kindler syndrome is a rare genodermatosis characterized by acral bullae formation, fusion of fingers and toes, and generalized as progressive poikiloderma (20).

\section{Cause}

Kindler syndrome is caused by genetic defects in the focal contact-associated protein, fermitin family homologue 1 (FFH1), encoded by the gene FERMT1 (known as KIND1) (7). Kindlin-1 is known to be expressed by the oral epithelium including the surface of the tongue (22).

Immunofluorescence, gene expression and cell biology studies have shown that kindlin-1 is expressed mainly in basal keratinocytes and plays a role in the attachment of the actin cytoskeleton via focal contacts to the extracellular matrix. Thus, Kindler syndrome is the first genodermatosis caused by a defect in actin-extracellular matrix linkage rather than the classic keratin-extracellular matrix linkage underlying the pathology of other inherited skin fragility disorders such as epidermolysis bullosa (1).

\section{Oral signs}

In individuals with Kindler Syndrome the oral manifestations can involve $(5,15,17,18,21,22)$ :

* increased tissue fragility of oral and perioral mucosa

* oral blistering 
Epidermolysis bullosa and Kindler syndrome - where is the border in oral manifestations

* chronic erosive gingivitis have been noted even in young children $(15,18,22)$.

* rapidly progressive form of periodontal disease (aggressive periodontitis) that can have its onset during teenage years $(18,21,22)$

* alveolar bone loss (22).

Other clinical features include:

* dysphagia

* esophageal and urethral strictures,

* ectropion, and

* an increased risk of mucocutaneous squamous cell carcinoma (15).

\section{Dental caries and salivary function}

The dentition of affected individuals appears to be normal. Similarly, the salivary function and risk of dental caries is unknown in this rare syndrome (22).

\section{DISCUSSION}

Epidermolysis bullosa (EB) is associated with diverse oral manifestations, which can potentially involve both hard and soft tissues, depending on the specific EB subtype (22).

In individuals with $\mathrm{EB}$ dental defects vary in the different subtypes of the disease but are most frequent in the autosomal recessive, scarring type of epidermolysis bullosa in which there may be delayed, or failure of, eruption. Dental abnormalities include fine or coarse pitting defects, or thin and uneven enamel which may also lack prismatic structure. The amelodentinal junction may be smooth (4)

Some authors mention that oral lesions and involvement of $\mathrm{EB}$ are most commonly observed in the dystrophic forms $(16,19)$. Dental abnormalities. such as anodontia, enamel hypoplasia, pitting of the enamel. neonatal teeth. and severe dental caries, have been variably associated with several of the ditterent types of epidermolysis bullosa, although recent studies have indicated that the prevalence of dental abnormalities is increased only with the junctional type $(16,19)$.

M.C. Serrano-Martínez et coworkers identified blister lesions in $92 \%$ of the patients with dystrophic $\mathrm{EB}$ at the time of exploration--the tongue being the most affected location. Microstomia and palatal atrophy were the most prevalent sequelae (100\%), while ankyloglossia, vestibular obliteration and lingual de- papillation were recorded in over $90 \%$. In $80 \%$ of the patients interincisal MOA was $<30 \mathrm{~mm}$ (severe microstomia) (19).

In dominant dystrophic types the oral manifestations are typically mild, with some gingival erythema and tenderness. Gingival recession and reduction in the depth of the buccal vestibule may be observed (16).

In Recessive dystrophic types the oral problems are also severe, bulla and vesicle formation is induced by virtually any food having some degree of texture, even with a soft diet the repeated cycles of scarring often result in microstomia and ankyloglossia. Similar mucosal injury and scarring may cause severe stricture of the esophagus. Because a soft diet is usually highly cariogenic. Carious destruction of the dentition at an early age is common (16).

Although Kindler syndrome is classified as a subtype of epidermolysis bullosa, it has distinct clinicopathological and molecular abnormalities $(12,13)$.

The clinical features of the syndrome have been annotated by different authors but the definite of criteria to confirm the diagnosis have not yet been generally accepted (9).

H. Shimizu et coworkers suggest that the bullous component of Kindler syndrome is distinct from dystrophic epidermolysis bullosa caused by mutations in the type VII collagen gene (20).

In the literature are described also a family casese with Kindler syndrome (10).

Risk for developing squamous cell carcinomas

Individuals with severe generalized recessive dystrophic EB and those with Kindler syndrome are at increased risk of oral squamous cell carcinoma formation and should therefore be extra vigilant in monitoring changes in oral ulcerations (22). There are reports of squamous cell carcinoma of the hard palate, lower lip, hand, bladder and larynx $(8,15,22,23)$.

A 57 year-old woman with diagnosis of Kindler Syndrome was observed, without family predilection. The initial diagnosis two years ago was Epidermolysis bullosa. Bulla formation started at birth on areas of the skin that received pressure mainly on the limbs. The patient referred to the Hospital with clinical and histological evidence of persistent squamous cell carcinomas of the lower lip and primary carci- 
noma of the hard palate. She also had bilateral, incomplete sindaktiliya poykilodermiya and progressive atrophy of the skin on the legs, neck and face. The woman was reoperation on the occasion of the primary skin verrucous squamous cell carcinoma. In 2007 was operated by reason of squamous cell carcinoma of the upper and lower lip. In 2008 was made electrocautery recurrence of cancer of the lower lip , as well as papillomas of the gingiva (6).

\section{Diagnosis}

EB Diagnosis requires histopathology as well as direct and indirect immunofluorescence studies (3).

The differential diagnosis of Kindler syndrome includes diseases like Bloom syndrome, Cockayne syndrome, dyskeratosis congenita, epidermolysis bullosa, Rothmund-Thomson syndrome and xeroderma pigmentosum (11).

\section{General treatment}

Treatment of epidermolysis bullosa varies with the type. EB Management includes corticosteroids, dapsone, azathioprine, mycophenolate mofetil, rituximab, and intravenous immunoglobulin (3).

For milder cases. no treatment other than local wound care may be needed. Sterile drainage of larger blisters and the use of topical antibiotics are often indicated in these situations. For the more severe cases. Intensive management with oral antibiotics may be necessary if cellulit is develops; despite intenive medical care, some patients dieas a result of infectious complications. The „mitten“ deformity of the hands, seen in recessive dystrophic epidermolysis bullosa, can be corrected with plastic surgery but the problem usually recurs after a period of time. and surgical intervention is required every 2 years on average. With esophageal involvement, dysphagia may be a significant problem. resulting in malnutrition and weight loss. Placement of a gastrostomy tube may be necessary at times. Patients with the recessive dystrophic forms are also predisposed to development of cutaneous squamous cell carcinoma. This malignancy often develops in areas of chronic ulceration during the second through third decades of life and represents a significant cause of death for these patients. In frequenly, the lingual mucosa of affected patients has been reported to undergo malignant transformation as well (16).
Treatment of Kindler syndrome is symptomatic and aimed at prevention. Patients should avoid sun exposure and use external photoprotection to limit the progression of poikiloderma and the risk of cutaneous carcinoma. Trauma should also be avoided to reduce blister formation, which can progress into disabling scars. Patients can expect to live relatively normal lives if they have close follow-up visits. Blisters tend to fade after childhood, usually without scars. However, poikiloderma and skin atrophy progresses over a period of time. Complications of Kindler syndrome, including neoplasms, may limit life expectancy (14).

\section{Oral therapy}

Management of the oral manifestatio ns also depends on the type of the disease. Oral lesions may be responsive to intensive topical corticosteroid therapy (3).

For patients who are susceptible to mucosal bulla formation dental manipulation should be kept to a minimum. To achieve this topical $1 \%$ neutral sodium fluoride solution should be administered daily to prevent dental caries. A soft diet that is as noncariogenic as possible, as well as atraumatic oral hygiene procedures. should be encouraged. Maintaining adequate nutrition for affected pati ents is critic al to ensure optimal wound healing. If dental restrative caris required the lips should be lubricated to minimize trauma. Injections for local anesthesia can usually be accom pl ished by depositing the anesthetic slowly and deeply wi thin the tissues. For extensive dental care endotracheal anesthesia may be performed without significant problems in most cases. Unfortunately. because of the genetic nature of these Diseases no cure exists. Genetic counseling of families is indicated and prenatal diagnosis is available (16).

\section{CONCLUSION}

There are many different manifestations of Kindler syndrome. Oral squamous cell carcinoma have been described a few times, presenting particularly on the lips and soft palate. These tumours are usually extremely aggressive. In lesions suspected for squamous cell carcinoma, close follow-up visits with complete anamnesis, physical examination and appropriate laboratory/radiological workup should be performed. The metastatic potential of the squa- 
mous cell carcinoma should prompt early diagnosis and management.

\section{REFERENCES}

1. Ashton GHS. Kindler syndrome. Clinical and Experimental Dermatology. 2004; 29(2):116-121.

2. Brush J, N Treister. Clinical oral medicine and pathology. Humana Press; 2010. p 66-68.

3. Burch J, H Fassihi, C Jones, et al. Kindler Syndrome: A New Mutation and New Diagnostic Possibilities. Arch Dermatol. 2006;142(5):620-624.

4. Cawson R, E Odell, S Porter. Essential of oral pathology and oral medicine. 7th ed. Elsevier Science Limired; 2002. p 26.

5. Chimenos KE, FR Fernández, J López, et al. Kindler syndrome: a clinical case. Med Oral. 2003;8(1):38-44.

6. Deliverska E, Hr Stoyanov. Kindler syndrome - review of the literature and clinical case. Savremena stomatologia. 2010;2. Bulgarian.

7. D‘Souza MA, RM Kimble, JR McMillan. Kindler syndrome pathogenesis and fermitin family homologue 1 (kindlin-1) function. Dermatol Clin. 2010;28(1):115-118.

8. Emanuel P, D Rudikoff, R Phelps. Aggressive Squamous Cell Carcinoma in Kindler Syndrome. SKINmed: Dermatology for the Clinician. 2006;5(6):305-307.

9. Fischer IA, J Kazandjieva, S Vassileva, et al. Kindler syndrome: a case report and proposal for clinical diagnostic criteria. Acta Dermatovenerol Alp Panonica Adriat. 2005;14(2):61-67.

10. Haber RM, WM Hanna. Kindler syndrome. Clinical and ultrastructural findings. Arch Dermatol. 1996;132(12):1487-1490.

11. Kaviarasan PK, Prasad PV, Shradda, et al. Kindler syndrome. Indian J Dermatol Venereol Leprol. 2005;71(5):348-350.

12. Lai-Cheong JE, A Tanaka, G Hawche, et al. Kindler syndrome: a focal adhesion genodermatosis. $\mathrm{Br} \mathrm{J}$ Dermatol. 2009;160(2):233-242.

13. Lai-Cheong JE, JA McGrath. Kindler syndrome. Dermatol Clin. 2010;28(1):119-124.

14. Langlois EC, D Hanna, M St-Amant, et al. Invasive squamous cell carcinoma of the hand in a patient with Kindler syndrome: Case report and literature review. Can J Plast Surg. 2010;18(3): 41-43.
15. Mizutani H, K Masuda, N Nakamura, et al. Cutaneous and Laryngeal Squamous Cell Carcinoma in Mixed Epidermolysis Bullosa, Kindler Syndrome Case Rep. Dermatol. 2012;4(2):133-138.

16. Neville B, D Damme, C Allen, et al. Oral \& maxillofacial pathology. 3rd ed. Philadelphia; Toronto: W.B. Saunders; 2008.

17. Ramesh CS, M Vikram, LS Nand, et al. International Journal of Dermatology. 2003;42(9):727-732.

18. Ricketts DN, CL Morgan, JM McGregor, et al. Kindler syndrome: a rare cause of desquamative lesions of the gingiva. Oral Surg Oral Med Oral Pathol Oral Radiol Endod. 1997;84(5):488-491.

19. Serrano-Martínez MC, JV Bagán, FJ Silvestre, et al. Oral lesions in recessive dystrophic epidermolysis bullosa. Oral Dis. 2003;9(5):264-268.

20. Shimizu H, M Sato, M Ban, et al. Immunohistochemical, ultrastructural, and molecular features of Kindler syndrome distinguish it from dystrophic epidermolysis bullosa. Archives of Dermatology, 1997;133(9):1111-1117.

21. Wiebe CB, G Petricca, L Häkkinen, et al. Kindler syndrome and periodontal disease: review of the literature and a 12-year follow-up case. J Periodontol. 2008;79(5):961-966.

22. Wright JT. Oral Manifestations in the Epidermolysis Bullosa Spectrum. Dermatol Clin. 2010;28(1): 159-164.

23. Wright JT, JD Fine, LB Johnson. Oral soft tissues in hereditary epidermolysis bullosa. Oral Surg Oral Med Oral Pathol. 1997;1(4):440-446. 